\title{
Adaptive Node Degree Adjustment Based Power Control Method for Wireless Sensor Networks
}

\author{
https://doi.org/10.3991/ijoe.v14i09.8683 \\ Huangshui Hu, Man Zheng $\left({ }^{\bowtie}\right)$, Bangcheng Zhang, Jinhui Qing \\ Changchun University of Technology, Changchun, China \\ 1839642147 @qq. com
}

\begin{abstract}
Properly adjusting the transmission power of the wireless sensor nodes has shown to be an effective approach to reduce energy consumption and to improve the network reliability. In this paper, an adaptive Node Degree adjustment based Power Control (NDPC) method for wireless sensor networks is proposed to dynamically adjust the transmission power of the nodes. In NDPC, each node is embedded with a fuzzy neural controller which is used to adjust the target node degree so as to control the communication range properly. The fuzzy neural controller consists of two Fuzzy Inference Systems (FIS). One adopts closed-loop feedback mechanism to adjust the target node degree according to the residual energy. The other adjusts the communication range by a self-learning neural network to control the transmission power based on the target node degree. Consequently, the actual energy consumption of the node is reduced while keeping the desired node degree. Several simulation experiments are conducted to evaluate the performance of NDPC, and the results show that NDPC can reduce the actual energy consumption as well as extend the network lifetime.
\end{abstract}

Keywords-wireless sensor networks, transmission power control, fuzzy logic, node degree, energy consumption

\section{Introduction}

Wireless Sensor Networks (WSNs) which consist of a certain number of tiny sensor nodes with integration of sensing and communication abilities have been widely investigated and deployed in a variety of application scenarios such as health-care, emergency response, environmental monitoring, and space exploration [1,2]. The impact from environments together with energy constraints of the nodes makes high energy efficiency as well as long lifetime a challenging task. Adjusting the transmission power of the individual nodes has shown to be an effective approach to reduce the energy consumption, decrease the amount of collisions as well as preserve the communication reliability $[3,4]$.

It has been experimentally shown that transmission power has significant impact on link quality $[4,5]$, which means a high transmission power level provides a good link quality at the expense of increasing the energy consumption, or vice versa. There- 
fore, most of the recent studies on transmission power control (TPC) employ link level strategies to maximize WSN lifetime and improve network performance $[3,6]$. Usually, the variation of link quality metrics such as Packet Reception Ratio(PRR), Reception Signal Strength Indicator(RSSI) and Link Quality Indicator(LQI) is used to adapt the transmission power [1,3,7-9]. However, link quality is susceptible to environmental inference and network dynamics. Accordingly, node degree has been widely used to adjust the transmission power because of its significant impact on signal inference, link reliability and latency times, and so on [7-10]. Many approaches are proposed to adjust sensor nodes' transmission power in order to obtain the desired node degree [8]. However, the neighbors' locations are usually required which is not always available in sensor nodes, so localized algorithms without assumption that location information is needed have been proposed [11]. What is more, intelligent control techniques such as fuzzy control is used for developing adaptation strategies on dynamics of WSNs and environment as well as constraints of the linear model, and the results show that these strategies can tolerate the uncertain interference and converge fast to keep the network stable, energy-efficient and communication-reliable [11-13].

In this paper, a novel transmission power control approach based on self-adaptive fuzzy controller is proposed to dynamically adjust the transmission power of the nodes, which is called NDPC. The fuzzy controller consists of two inference engines, one is responsible for node degree adjustment, and the other is responsible for the node transmission power adjustment, so as to adjust the transmission power adaptively according to the residual energy of the node. Moreover, the parameters of the first inference engine are from an if-then rule base, and the parameters of the second inference engine are obtained by a neural network with a training data set. Through the closed-loop feedback processes, the fuzzy controller can be adapted to the changes of node degree with respect to the residual energy, accordingly, control the transmission power of the nodes.

The remainder of this paper is organized as follows. The related works are introduced in Section 2. The proposed NDPC is presented in Section 3. Section 4 provides the simulation and performance evaluation of NDPC. Finally, section 5 concludes the paper.

\section{$2 \quad$ Related works}

Usually, transmission power control approaches concentrate on maintaining the lowest transmission power level compatible with the acceptable link quality, which are categorized into three major groups network level, node level and link level $[5,7]$. In network level solutions [14,15], a single transmission power for the whole network is adopted to achieve course tuning of power control, which inevitably leads to high energy consumption as well as not making full use of the configurable transmission power provided by radio hardware. In link level solutions, transmission power is adjusted to provide a certain level of the link quality so as to maximize WSN lifetime [3,16-19]. In [3], a lightweight algorithm for adaptive transmission power control in 
WSN is proposed. In this algorithm, each node builds a model for each of its neighbors to describe the correlation between transmission power and link quality, and with this model, a feedback-based transmission power control is used to dynamically maintain individual link quality over time. In [18], a transmission power control scheme is proposed to improve the WSN energy efficiency, in which the minimum transmission power level is used for data transmission on each link that ensures a predetermined target packet error probability whereas control packets are transmitted using the maximum power level. In [19], an approach to continuously monitor link quality for multiple transmission power levels is proposed which enables the selection of lowest transmission power level that achieves the target reliability level. However, extensive empirical studies have shown that link quality is so largely influenced by the time and environment $[7,20]$, that is non-deterministic to real world deployments. In node level solutions $[11,16,17]$, an optimal transmission power is selected to maintain the communication between pair of nodes or among a node and all its neighbors, in order to reduce energy consumption while keeping communication reliability. In [22], a novel Fuzzy-logic Topology Control (FTC) is proposed to achieve any desired average node degree by adaptively changing communication range, thus improving the network connectivity. However, the WSN is inevitably dynamic since the nodes will be quit or added to the network, and the residual energy ignorance will undoubtedly lead to unbalanced energy consumption with node premature death.

Furthermore, WSNs often have to deal with some uncertainty of ambiguity which can be described by fuzzy logic $[11,13,23]$. Fuzzy logic is characterized by model free, which means that it can dispose of accidental interference and uncertain factors so as to be employed in transmission power control with dynamic and unpredictable environments. In [11], a close-loop control system using fuzzy control theory is applied upon WSN, in which each node acts as a controller and its neighbor nodes as a plant, and the control action is depend on the number of its neighbor nodes. With the control system, uncertain interference in the network can be efficiently overcome and energy consumption can be reduced significantly. In [2], a self-adaptive system through two feedback control loops based on fuzzy control is proposed. In this system, the primary feedback control loop adjusts the node transmission power considering both its real and targeted number of neighbors, and the secondary feedback control loop manages the node targeted number of neighbors considering the battery level. The simulation showed that the self-adaptive system allows the nodes in the network to achieve a balance between a good enough power saving while keeping a high reliability of communications. Moreover, a novel localized fuzzy logical approach to adaptively control the transmission power of each node is proposed in [22] so as to achieve the desired node degree. Especially, in this approach the fuzzy logic controller is constructed from the training data set instead of designing membership functions and if-then rules in traditional methods in order to facilitate the design process. Accordingly, it is proved to be accurate, stable and has short settling time. However, ignoring the variety of energy will make nodes with lower energy transmit data packets with higher power level so as to enter premature death state. Besides, lack of learning ability on the existing data set inevitably restricts the network scalability and applications in different environment. 
In this paper, the fuzzy logic system serves as two inference engines for each sensor node to modify its transmission power according to its residual energy while keeping the desired node degree. Moreover, unlike other fuzzy logic control methods for WSNs, the parameters of the first inference engine are obtained by a neural network with a training data set, and the parameters of the second inference engine are acquired by if-then rules. Therefore, our proposal is more flexible to deal with network dynamics while keeping balanced energy consumption.

\section{Design of NDPC}

With respect to the power saving and reliability of the wireless sensor networks, it seems an impertinent approach to only apply a single fuzzy logic system when adjusting transmission power. Therefore, a neural fuzzy controller based system which consists of two inference engines is designed to adaptively adjust the transmission power according to the residual energy of the node with desired node degree. By means of applying the self-learning neural network, the first inference engine (FIS1) can learn from training data set to control transmission power. And by using the rules from domain experts, the second inference engine (FIS2) can adjust the targeted node degree according the residual energy. The architecture of NDPC is illustrated in Fig.1.

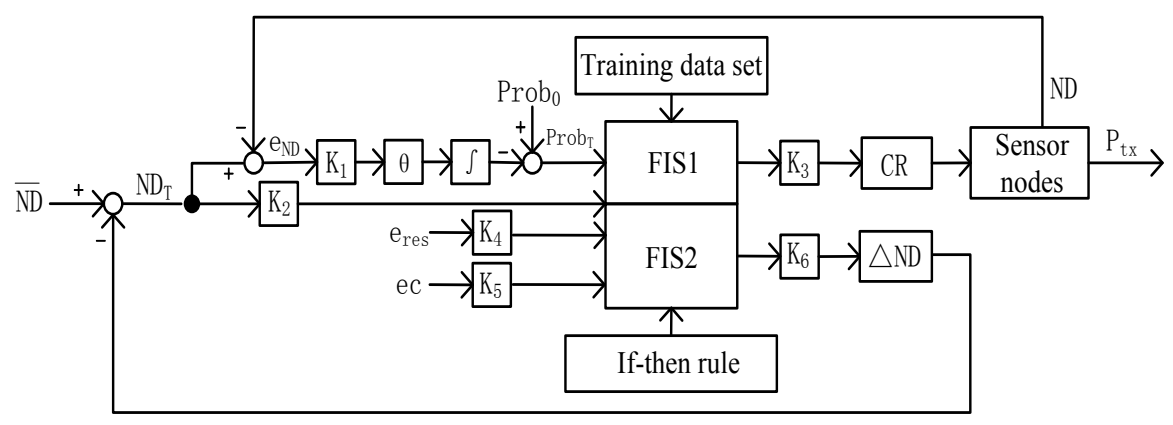

Fig. 1. Architecture of NDPC

\subsection{Input and output}

As seen from Fig.1, NDPC consists of two inference engines which have a common input denoted by desired node degree $\overline{N D}$. Also $\overline{N D}$ is used to calculate the targeted node degree $N D_{T}$ by adding the variation to be applied $\Delta N D$ estimated by FIS2 based on the residual energy. In addition, the other input of FIS1 is the probability $\operatorname{Prob}$ that a node has degree $\overline{N D}$. Moreover, adjusting the transmission power is a very common capability in many sensor nodes, hence, the output of FIS1 is the communication range (or equivalently transmission power) $C R$. On the other hand, the 
second input of FIS2 is the residual energy deviation $e_{\text {res }}$, the third input is the ratio of residual energy and the deviation of communication range, that is $e c=e_{\text {res }} / e_{\mathrm{cr}} . K_{1}$ 、 $K_{2}, K_{3}, K_{4}, K_{5}$ and $K_{6}$ in Fig. 1 are scale factors.

The probability that the node degree is at least bigger than $k$ is given by Eq.(1) as in [22].

$$
P(N D \geq k)=f(k, r)=1-\sum_{N=0}^{k-1} \frac{\left(\rho \pi r^{2}\right)^{N}}{N !} e^{-\rho \pi r^{2}}
$$

where $r$ is the node communication radius, $\rho=\frac{n}{A}$ is node density, $n$ is the total number of nodes in the network, and $A$ is the area of the field of interest. As illustrated in Fig.1 and Eq.(1), the inputs of FIS1 are $\overline{N D}$ and Prob, and the output is $P_{t x}$. Given the above parameter values, $C R \in\left\{r_{1}, r_{2}, \ldots, r_{j}\right\}$, and $\overline{N D} \in\left\{k_{1}, k_{2}, \ldots, k_{m}\right\}$, then $\operatorname{Prob}=f(\overline{N D}, C R)$ can be calculated from Eq. (1). The training data set $T$ is a $s \times 3$ matrix in the form of [ $\overline{N D}, \operatorname{Prob}, C R]$, where $s=m \times j$.

For the energy model, the first order radio model described in [22] is adopted to calculate the residual energy deviation. For the pair of nodes $i, j$, the energy consumed in the transmit state with $l$-byte and receive state with $m$-byte for a linear communication as a function of the distance $d_{i j}$ is given by Eq. (2).

$$
\Delta E=E_{I}-8 \times\left(l E_{e}+l E_{r} d_{i j}^{4}+m E_{e}\right)
$$

Where $E_{I}$ is the residual energy before the transmission, and $E_{e}$ is the energy consumed by the corresponding electronic circuits, and $E_{r}$ is the energy consumed by the power amplifier. Like in [22], the parameters of $E_{e}, E_{r}$ are both assumed to be 50 nj / bit.

\subsection{The first inference engine}

FIS1 is a self-learning fuzzy neural network, which combines the advantages of fuzzy control in learning complex and nonlinear systems with the learning ability of artificial neural networks. The system learning process uses gradient descending back propagation algorithm to adjust the condition parameters and the conclusion parameters. Its structure is shown in Fig.2. Through training and learning of data sets, it can achieve the ideal that output communication range can be output with arbitrary input target node degree and the desired probability. And the network layers are as follows: 


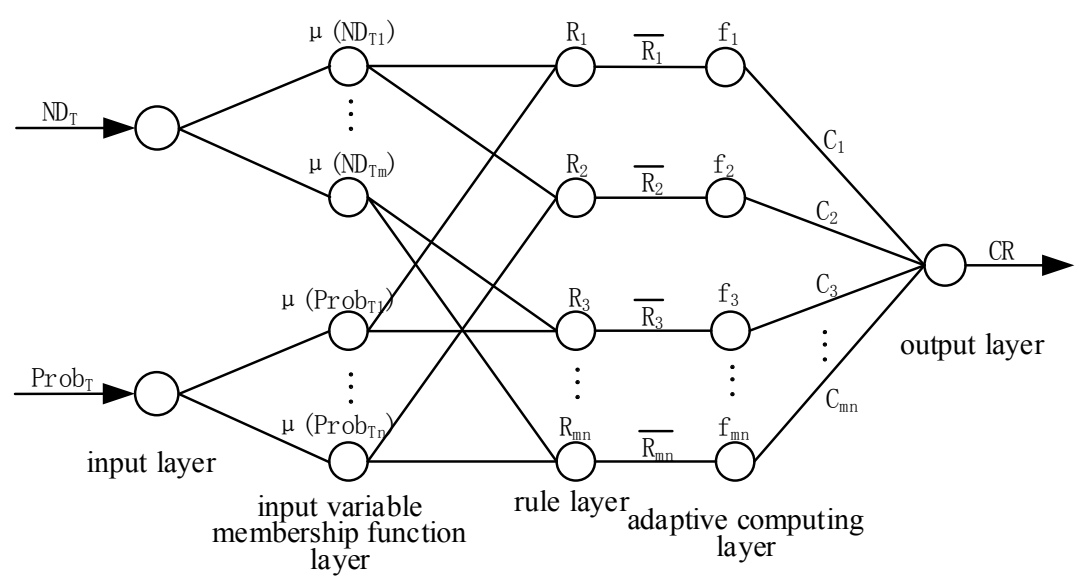

Fig. 2. Architecture of FIS1

(1) Input layer: The network has two inputs, namely $\overline{N D}$ and Prob.

(2) Membership functions layer: According to the collected data of $\overline{N D}$, Prob and $C R$, the training data set $[\overline{N D}, \operatorname{Prob}, C R]$ is obtained which is used for training the model. For the $j_{t h}$ data set, the bell-shaped function is used to fuzzy the input variables. Membership function of each variable is given by Eq. (3).

$$
\left\{\begin{array}{l}
\mu\left(\overline{P R R}_{i}\right)=\exp \left(-\left(\overline{P R R}^{j}-c_{j}^{i}\right)^{2} / b_{j}^{i}\right) \\
\mu\left(\operatorname{Prob}_{i}\right)=\exp \left(-\left(\operatorname{Prob}^{j}-c_{j}^{i}\right)^{2} / b_{j}^{i}\right)
\end{array} \quad(i=1,2)\right.
$$

Where $i$ is the number of fuzzy subsets, and $c_{j}^{i}, b_{j}^{i}$ are the center and width of membership functions.

(3) Rule layer: The fuzzy operation is performed on the data. And the output is the normalized value of each neuron input product, namely the normalization of the excitation intensity of each rule, which is shown as follows:

$$
\begin{gathered}
R_{j}=\mu_{\mathrm{ND}_{\mathrm{T} a}} \cdot \mu_{\mathrm{Prob}_{\mathrm{T} b}} \\
\overline{R_{j}}=\frac{R_{j}}{R_{1}+R_{2}+\mathrm{K}+R_{m n}}
\end{gathered}
$$

Where $R_{j}$ means rule $j, a \in\{1,2, \mathrm{~L}, m\}, b \in\{1,2, \mathrm{~L}, n\}, j=1,2,3, \mathrm{~L}, m n$.

(4) Adaptive computing layer: This layer combined with the control rules to complete the adaptive computing, and the output of each rule can be calculated. Node output result is as follows: 


$$
C_{j}=\overline{R_{j}} f_{j}=\overline{R_{j}}\left(p_{j} \cdot \mathrm{ND}_{\mathrm{T}^{j}}+q_{j} \cdot \operatorname{Prob}_{\mathrm{T}}^{j}+r_{j}\right)
$$

Where $\left\{p_{j}, q_{j}, r_{j}\right\}$ are the node's parameters.

(5) Output layer: The total network training output represents the node communication range value, which is predicted from the expected energy consumption and packet size of the input node, and the result is the sum of the four outputs in the adaptive computation layer:

$$
\mathrm{CR}=C_{1}+C_{2}+C_{3}+\mathrm{L}+C_{m n}
$$

(6) Learning process: given the learning error function as follows:

$$
e=\frac{1}{2}\left(\mathrm{CR}_{d}-\mathrm{CR}_{c}\right)^{2}
$$

Where $\mathrm{CR}_{d}, \mathrm{CR}_{c}$ are the desired and real communication range of the node. Then the system adjusts the parameters weight vale $\omega$, the center and width values $c, b$ of the membership function to achieve the desired effect by continuous learning. And the parameters can be obtained by Eq. (8) (9) (10) respectively.

$$
\begin{gathered}
\omega(k)=\omega(k-1)-\alpha \frac{\partial e}{\partial \omega} \\
c(k)=c(k-1)-\alpha \frac{\partial e}{\partial c} \\
b(k)=b(k-1)-\alpha \frac{\partial e}{\partial b}
\end{gathered}
$$

Where $k$ is the number of learning process, and $\alpha$ is the learning rate.

\subsection{The second inference engine}

FIS2 uses Mamdani-type fuzzy controller to adaptively adjust the desired node degree through the closed-loop feedback mechanism to obtain the optimal target node degree. The input variables of FIS2 are the ratio of the target node degree $\mathrm{ND}_{\mathrm{T}}$, the remaining energy deviation $e_{\text {res }}$ and the ratio $\mathrm{ec}=e_{\text {res }} / e_{\mathrm{cr}}$ of the remaining energy deviation and the communication range deviation, and the output variable is the expected node deviation $\triangle$ ND. FIS2 mainly consists of several parts like fuzzification, fuzzy rule, and defuzzification which are described in detail next.

(1) Fuzzification

The clear value of the target node degree $\mathrm{ND}_{\mathrm{T}}$ of the input variable is $[0,1,2,3$, 4], and the corresponding fuzzy language variables are "low", "low_middle", "middle", "middle_high" and "high". Similarly, the clear value of the residual energy devi- 
ation $e_{\text {res }}$ of the input variables is $[0,1,2]$, and the corresponding fuzzy language variables are "small", "middle" and "large"; and the clear value of the ratio ec of the remaining energy deviation and the communication range deviation is $[-1,0,1]$, and the corresponding fuzzy language variables are "negative", "O" and "positive"; the clear variable of desired node degree deviation $\triangle \mathrm{ND}$ of the output variable is $[-2,-$ $1,0,1,2]$, and its corresponding fuzzy language variables are "D2S", "D1S", "HOLD", "U1S", "U2S ". Besides, "low", "high", "small", "large", "negative", "positive", "D2S" and "U2S" are the trapezoidal membership functions, while "low_middle", "middle", "middle_high", " O "," D1S "," HOLD ", and" U1S "are triangular membership functions. Input and output variables membership functions are shown in Fig.3.

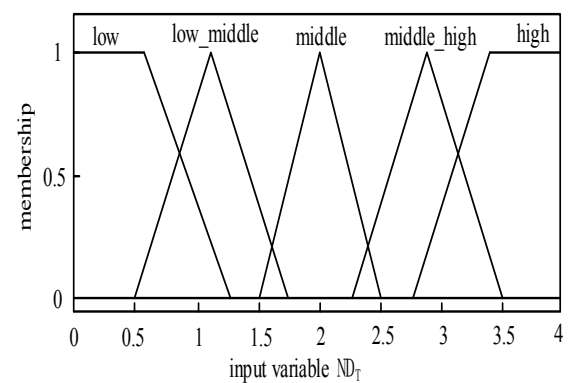

Fig.3(a) Membership functions for $\mathrm{ND}_{\mathrm{T}}$

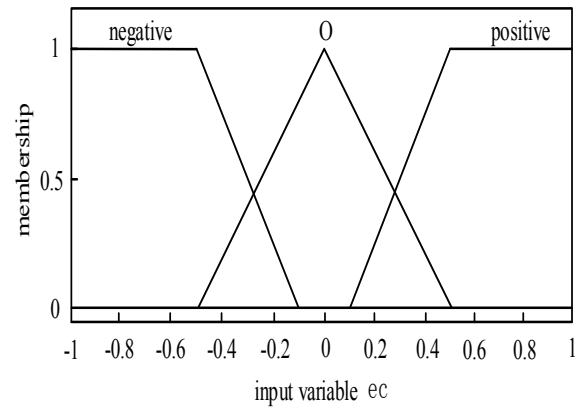

Fig.3(c) Membership functions for ec

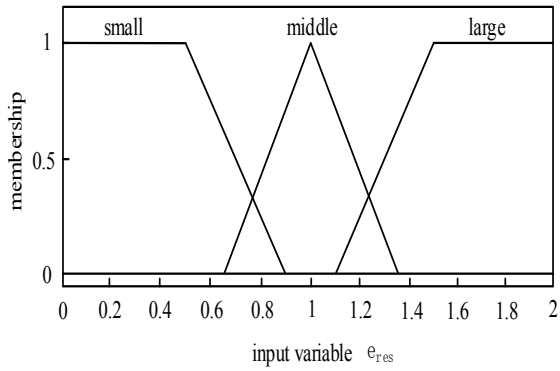

Fig.3(b) Membership functions for $e_{\text {res }}$

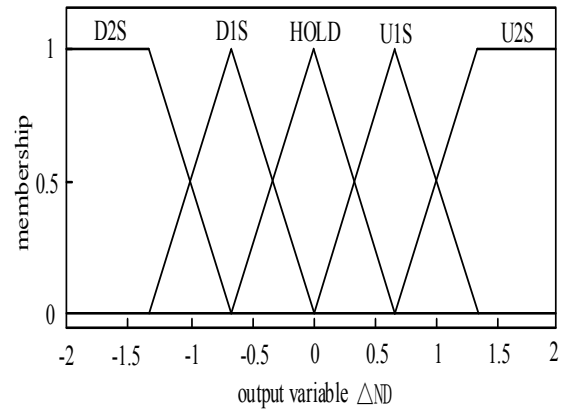

Fig.3(d) Membership functions for $\Delta \mathrm{ND}$

Fig. 3. Membership functions for inputs and outputs

Fuzzy rules and defuzzification. Using the membership functions given above, the input sharpness values can be fuzzified to the corresponding fuzzy language variables. Then these fuzzy language variables can output corresponding fuzzy language variables after being processed by if-then rules in the fuzzy reasoning system. Fuzzy rules are shown in Table.1. 
Table 1. Fuzzy rules

\begin{tabular}{|c|c|c|c|c|}
\hline \multirow[t]{2}{*}{ No. } & \multicolumn{3}{|c|}{ Input } & \multirow{2}{*}{$\begin{array}{c}\text { Output } \\
\Delta \mathrm{ND}\end{array}$} \\
\hline & $\mathrm{ND}_{\mathrm{T}}$ & $e_{\text {res }}$ & $e c$ & \\
\hline 1 & low & small & negative & HOLD \\
\hline 2 & low & small & $\mathrm{O}$ & HOLD \\
\hline 3 & low & small & positive & D1S \\
\hline 4 & low & middle & negative & D1S \\
\hline 5 & low & middle & $\mathrm{O}$ & D1S \\
\hline 6 & low & middle & positive & D2S \\
\hline 7 & low & large & negative & D1S \\
\hline 8 & low & large & $\mathrm{O}$ & D2S \\
\hline 9 & low & large & positive & $\mathrm{D} 2 \mathrm{~S}$ \\
\hline 10 & low_middle & small & negative & HOLD \\
\hline 11 & low_middle & small & $\mathrm{O}$ & D1S \\
\hline 12 & low_middle & small & positive & $\mathrm{D} 2 \mathrm{~S}$ \\
\hline 13 & low_middle & middle & negative & HOLD \\
\hline 14 & low_middle & middle & $\mathrm{O}$ & D1S \\
\hline 15 & low_middle & middle & positive & D1S \\
\hline 16 & low_middle & large & negative & HOLD \\
\hline 17 & low_middle & large & $\mathrm{O}$ & HOLD \\
\hline 18 & low_middle & large & positive & D1S \\
\hline 19 & middle & small & negative & HOLD \\
\hline 20 & middle & small & $\mathrm{O}$ & HOLD \\
\hline 21 & middle & small & positive & HOLD \\
\hline 22 & middle & middle & negative & U1S \\
\hline 23 & middle & middle & $\mathrm{O}$ & HOLD \\
\hline 24 & middle & middle & positive & HOLD \\
\hline 25 & middle & large & negative & U1S \\
\hline 26 & middle & large & $\mathrm{O}$ & U1S \\
\hline 27 & middle & large & positive & HOLD \\
\hline 28 & middle_high & small & negative & U1S \\
\hline 29 & middle_high & small & $\mathrm{O}$ & HOLD \\
\hline 30 & middle_high & small & positive & HOLD \\
\hline 31 & middle_high & middle & negative & U2S \\
\hline 32 & middle_high & middle & $\mathrm{O}$ & U1S \\
\hline 33 & middle_high & middle & positive & U1S \\
\hline 34 & middle_high & large & negative & $\mathrm{U} 2 \mathrm{~S}$ \\
\hline 35 & middle_high & large & $\mathrm{O}$ & U2S \\
\hline 36 & middle_high & large & positive & U1S \\
\hline 37 & high & small & negative & U1S \\
\hline 38 & high & small & $\mathrm{O}$ & HOLD \\
\hline 39 & high & small & positive & HOLD \\
\hline
\end{tabular}




\begin{tabular}{|c|c|c|c|c|}
\hline 40 & high & middle & negative & U2S \\
\hline 41 & high & middle & O & U1S \\
\hline 42 & high & middle & positive & U1S \\
\hline 43 & high & large & negative & U2S \\
\hline 44 & high & large & O & U2S \\
\hline 45 & high & large & positive & U1S \\
\hline
\end{tabular}

Then, the centroid method is adopted to defuzzify the output in order to get the clear output value, which is given by Eq. (12).

$$
\Delta \mathrm{ND}=\frac{\sum_{i=1}^{n} x_{i} \mu_{\Delta \mathrm{ND}}\left(x_{i}\right)}{\sum_{i=1}^{n} \mu_{\Delta \mathrm{ND}}\left(x_{i}\right)}
$$

\subsection{NDPC algorithm}

The fuzzy neural controller designed above can be constructed with ANFIS, an adaptive fuzzy neural system tool in MATLAB, besides, evalfis is a function of the fuzzy inference system in MATLAB, representing FIS1 of NDPC. FIS2 is represented by fis $2\left(\mathrm{ND}_{\mathrm{T}}, e, e c\right)$. A node in the network initially broadcasts a HELLO message in the maximum communication range $r_{\max }$, which contains its unique id, to collect its neighbor information and obtain its actual node degree and remaining energy value. Then the data can be written into the fuzzy neural controller to adaptively control the communication range. The algorithm pseudo code is shown as follows.

It is given that:

1: training set: $T=\left[\mathrm{ND}_{\mathrm{T}}, \operatorname{Prob}_{\mathrm{T}}, \mathrm{CR}\right]$;

2: the maximum communication range of the node: $r_{\max }$;

3: the expected node degree: $\overline{\mathrm{ND}}$, the deviation of the node degree: $e_{\mathrm{ND}}$; the threshold of the node deviation: $\overline{e_{\mathrm{ND}}}$;

4: initial energy: $E_{\text {init }}$;

5: the number of cycles: rounds $=0$;

6: $\theta_{0}=0.02 \square$ Prob $_{0}=0.8$;

The algorithm starts:

7: Obtain the fuzzy neural controller by ANFIS(T);

8: $\mathrm{CR}_{u} \Leftarrow r_{\max }$;

9: $e_{\text {res }}($ rounds $)=E_{\text {init }}$;

10: do $\square$

11: Broadcast HELLO message with current $\mathrm{CR}_{u}$;

12: For messages received from other nodes, store the ID of its neighbors;

13: computing the actual node degree ND in the neighbor list and remaining energy $e_{\text {res }}$ (rounds) when nodes transmit in communication range $\mathrm{CR}_{u}$; 
14: computing the target node degree: $\mathrm{ND}_{\mathrm{T}}=\overline{\mathrm{ND}}-\Delta \overline{\mathrm{ND}}$ and the deviation of the node degree $e_{\mathrm{ND}}=\mathrm{ND}_{\mathrm{T}}-\mathrm{ND}$;

15: rounds $+=1$; computing the remaining energy deviation $e=e_{\text {res }}$ (rounds) $-e_{\text {res }}$ (rounds +1$)$;

16: if $e_{\mathrm{ND}}>0$ then

17: $\quad \theta=\frac{\theta_{0}}{2}$

18: else

19: $\theta=\theta_{0}$;

20: end if

21: $\quad \operatorname{Prob}_{\mathrm{T}} \Leftarrow \operatorname{Prob}_{0}-\int \theta \cdot e_{\mathrm{ND}}$;

22: $\quad$ fis $2\left(\mathrm{ND}_{\mathrm{T}}, e, \mathrm{ec}\right)$;

23: $\quad \mathrm{CR}_{u} \Leftarrow$ evalfis $\left(\mathrm{ND}_{\mathrm{T}}, \operatorname{Prob}_{\mathrm{T}}\right)$;

24: $\quad$ while $\left(e_{\mathrm{ND}}>\overline{e_{\mathrm{ND}}}\right)$;

25: end;

\section{Simulation results}

In order to verify the performance of NDPC algorithm, ATPC[5], FCTP[12], FTC[22] and NDPC are compared towards the average energy consumption, packet rate and network lifetime under MATLAB simulation environment. MICA2 is chosen as the simulation node. It is given that all nodes are randomly distributed in an area of $200 \mathrm{~m} \times 200 \mathrm{~m}$, the coordinates of the sink node are $(100,100)$, the initial energy of nodes is $1 \mathrm{~J}$, the number of transmission data bits is $4000 \mathrm{bits}$, the packet size is 500 bytes and the control packet size is 25 bytes. Besides, the scale factors are based on the real parameters of MICA2. Set $K_{1}=6, K_{2}=2, K_{3}=5, K_{4}=0.5, K_{5}=4$, $K_{6}=3$. The simulation results are the average of 50 experimental results.

Firstly, the relationship in the algorithm between the nodes' average total energy consumption and the network capacity is tested. The average total energy consumption of the nodes is the average of the total energy consumption of all the nodes in the network. The result is shown in Fig.4.

As it can be seen from the Fig.4, the average total energy consumption of FTC is the highest, which can account for it is that every time the node adjusts the transmit power, it needs to go through 10 rounds of fuzzy neural calculations. However, FCTP requires only 4 rounds of fuzzy control for each adjustment, so the average total energy consumption is lower than FTC. Besides, the algorithm considers not only the difference of the expected node degree and the actual node degree of the network but also the step length of the fuzzy control adjustment, so the transmit power can be quickly adjusted by the fuzzy controller, which can explain why FCTP converges faster than FTC. However, when the number of nodes is less than 200, the average total energy consumption of FCTP is lower than that of NDPC. This is because com- 


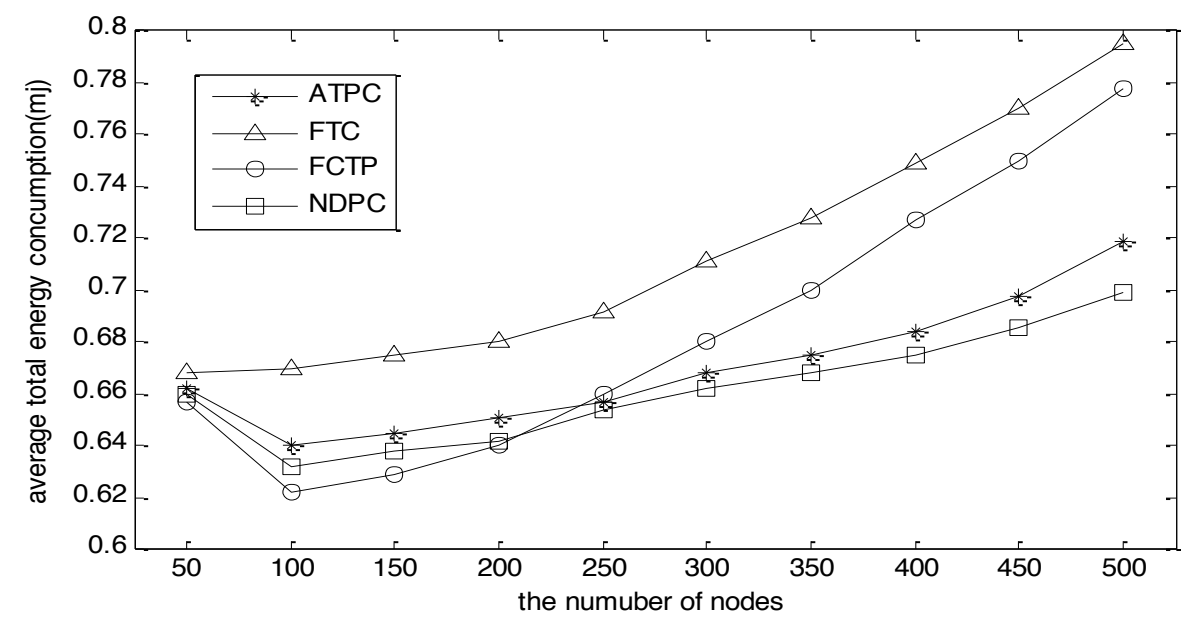

Fig. 4. Average total energy consumption of the nodes versus the number of nodes

pared with FCTP, NDPC uses two fuzzy inference systems in the adjustment process.Although the adjustment value of the adjusted transmission power is better, the calculation is slightly larger than FCTP. When the number of nodes is small, the average total energy consumption of NDPC is less than that of FCTP. With the expansion of network size, FCTP has too many premature death nodes and the overall performance declines. NDPC considers the remaining energy of the node to adjust its transmitting power, resulting in more balanced energy consumption. ATPC adjusts the transmit power of nodes according to the feedback of link quality to maintain high packet rate. Compared with FTC, FCTP and NDPC, ATPC can adjust faster and can reduce the average total network energy consumption. However, maintaining a high packet-receiving rate also requires a larger transmitting power. Without consideration of the remaining energy, in order to maintain a high packet-rate, nodes with a low residual energy will die early because of using a larger power transmission. In the adjustment of the transmitting power, NDPC not only adjusts the step size but also considers the residual energy of the node, thus effectively improving the network energy utilization efficiency.

Next, the network packet rate (PRR) has been tested with 300 nodes. The result of the test is shown in Fig.5. As can be seen from the figure, since the FTC only considers the target node degree of the node and ignores the link quality, the reliability of the link between the nodes and the PRR both decreases rapidly with the increase of the number of running cycles. However, FCTP takes the adjustment of the step length into account and adopts a non-linear method to adjust the transmit power of nodes. This method has a fast convergence, and it is easy to maintain the corresponding link quality. So the decrease of PRR is slower than that of FTC. The ATPC algorithm mainly considers the link quality in the process of adjusting the transmit power, and its feedback regulation goal is to maintain a certain packet reception rate, so the PRR can be hold at a high level. However, as the number of running rounds increasing, the node energy decreases, resulting in a decrease in PRR. The NDPC algorithm consid- 
ers not only the residual energy of nodes but also the adjustment of step size. It does improve the robustness. Therefore, the PRP of NDPC can stay at a high and steady state.

Finally, the network life cycle has been tested based on the survival nodes with 300 nodes. The result is shown in Fig.6. Because the other three algorithms do not consider the remaining energy of nodes, it is easy to cause nodes with low remaining energy dying early at a high transmit power. FTC adjusts the transmission power through the learning of the data, it is not easy to obtain the optimal transmission power, and the adjustment is more frequent, which leads to a waste of the most energy. FCTP adjusts the adjustment step size according to the degree of node deviation, reducing the number of adjustment, which reduces the energy consumption compared with FTC. However, the APTC adjusts the transmit power by means of link quality feedback, so that it can be quickly adjusted to meet a certain packet receiving rate. This method has a faster adjustment speed and lower communication energy consumption. However, NDPC can adjust its transmission power through data learning, it adaptively change the step size to accelerate the convergence. Considering the remaining energy of the node, NDPC avoids nodes with low remaining energy from transmitting data with high power, thus prolonging the network life cycle.

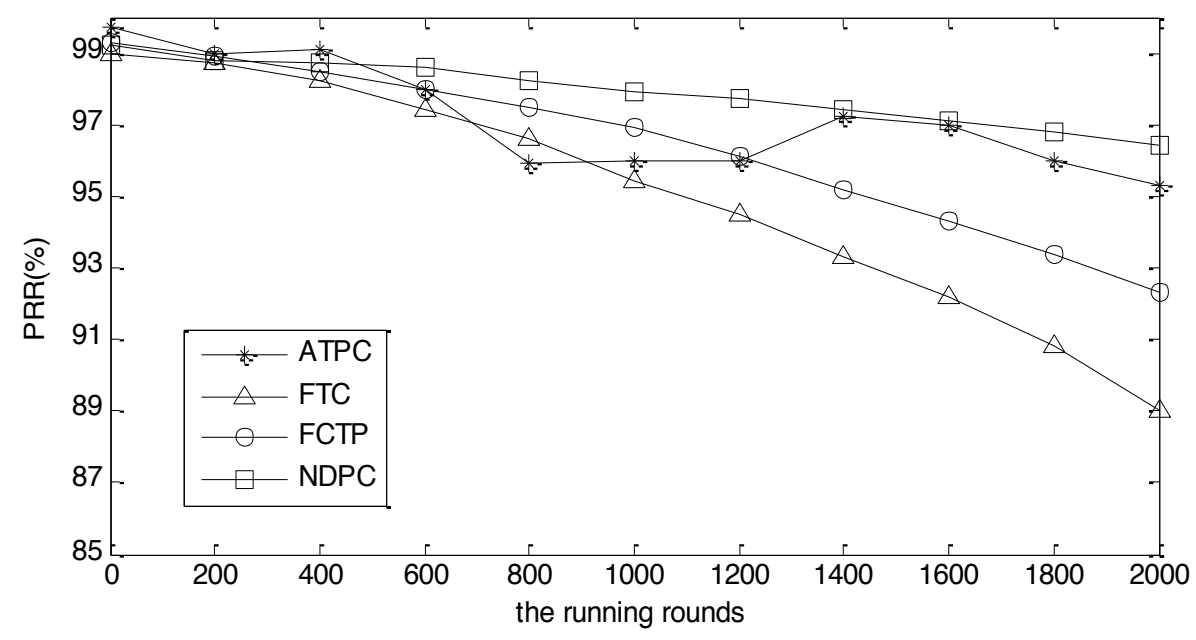

Fig. 5. Average PRR versus the running rounds 


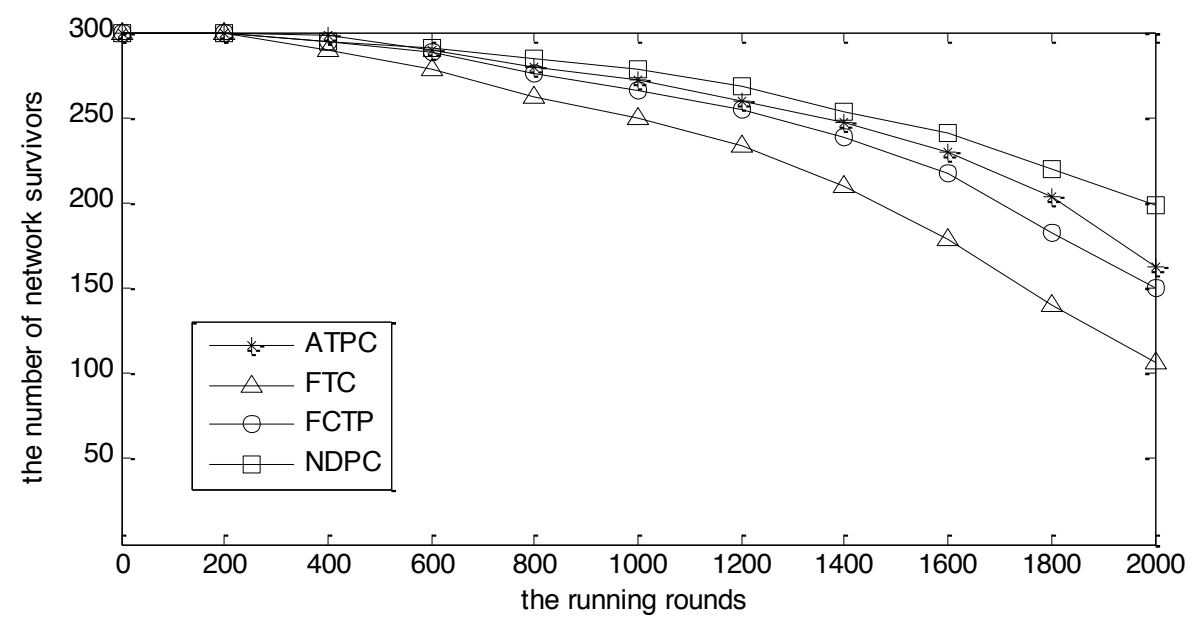

Fig. 6. The number of network survivors versus the running rounds

\section{Conclusion}

In this paper, a power control method based on fuzzy neural controller (NDPC) has been proposed. It consists of two fuzzy inference systems, and it adaptively adjusts the target node degree according to the residual energy of nodes to control the node transmission power. The simulation results show that NDPC has a better performance in terms of total node average energy consumption, packet rate and network life cycle than APTC, FTC and FCTP, which can effectively reduce node energy consumption and extend network life cycle.

\section{Acknowledgment}

This work was supported by Jilin Provincial Development and Reform Commission Project (2018C039-2); Science and technology program of Jilin provincial science and Technology Department (20150204073GX); Jilin Provincial Department of science and technology pilot project (20160312002ZG).

\section{$7 \quad$ References}

[1] TAHAR E, AMIRA Z. Efficient measurement of temperature, humidity and strain variation by modeling reflection Bragg grating spectrum in WSN[J]. Optik - International Journal for Light and Electron Optics, 2017, 135:454-462.

[2] JAVIER L, RUBEN R, FENG B, et al. Evolving privacy: From sensors to the Internet of Things[J]. Future Generation Computer Systems, 2017, 75: 4657. https://doi.org/10.1016/j.future.2017.04.045 
[3] Xue Z. Topology Control for Wireless Sensor Networks[J]. Journal of Software, 2007, 18(4): 943-954. https://doi.org/10.1360/jos180943

[4] PANTAZIS N A, VERGADOS D. A survey on power control issues in wireless sensor networks[J]. IEEE Communications Surveys \& Tutorials. 2007, 9(4): 86107. https://doi.org/10.1109/COMST.2007.4444752

[5] SHAN L, FEI M, ZHANG J B, et al. ATPC: Adaptive Transmission Power Control for Wireless Sensor Networks[J]. ACM Transactions on Sensor Networks, 2016, $12(1): 6$.

[6] HUSEYIN U Y, BULENT T. Transmission power control for link-level handshaking in wireless sensor networks[J]. IEEE Sensors Journal. 2016, 16(2): 561576. https://doi.org/10.1109/JSEN.2015.2486960

[7] HUSEYIN C, KEMAL B, BULENT T, et al. The impact of transmission power control strategies on lifetime of wireless sensor networks[J]. IEEE Transactions on Computers. 2014, 63(11): 2866-2879. https://doi.org/10.1109/TC.2013.151

[8] Son B H, Kim K J, Choi Y M. Active-margin transmission power control for wireless sensor networks. International Journal of Distributed Sensor Networks. 2014, 2014(1): 1-7.

[9] LIU T. Distributed power control mechanism based on utility model in wireless sensor networks [J]. electronic journal, 2016, 44(02): 301-307.

[10] GUO Z Q, WANG Q, LI M H, et al. Fuzzy Logic Based Multidimensional Link Quality Estimation for Multi-Hop Wireless Sensor Networks[J]. IEEE Sensors Journal, 2013, 13(10):3605-3615. https://doi.org/10.1109/JSEN.2013.2272054

[11] KUUBISCH M, KARL H, WOLISZ A, et al. Distributed algorithm for transmission power control in wireless sensor networks[C]. Wireless Communications and Networking, WCNC 2003. USA, New Orleans, IEEE, 2003(1): 558-563.

[12] Zhang J, Chen J, Sun Y. Transmission power adjustment of wireless sensor networks using fuzzy control algorithm[J]. Wireless Communications \& Mobile Computing, 2009, 9(6):805-818. https://doi.org/10.1002/wcm.630

[13] VICENTE H D, JOSE F M, NESTOR L M, et al. Self-adaptive strategy based on fuzzy control systems for improving performance in wireless sensors networks[J]. ACM Transaction on Sensor Networks. 2015, 15(9): 24125-24142.

[14] NARAYANASWAMY S, KAWADIA V, and SREENIVAS R S, et al. Power control in Ad-Hoc networks: theory, architectur, algorithm and implementation of the COMPOW protocol[C]. In Proceedings of the European Wireless Conference. 2002, PP: 156-162.

[15] SANTI P, BLOUGH D M, BOSTELMANN H. The critical transmitting range for connectivity in sparse wireless ad-hoc networks[J]. IEEE Transactions on Mobile Computing.2003, 2(1): 25-39. https://doi.org/10.1109/TMC.2003.1195149

[16] Lim G. Link Stability and Route Lifetime in Ad-hoc Wireless Networks[C]. IEEE Parallel Processing Workshops International Conference, 2002:116-123.

[17] Senouci M R, Mellouk A, Senouci H, et al. Performance evaluation of network lifetime spatial-temporal distribution for WSN routing protocols[J]. Journal of Network \& Computer Applications, 2012, 35(4):1317-1328. https://doi.org/10.1016/j.jnca.2012.01.016

[18] Yanagihara K, Taketsugu J, Fukui K, et al. A Clustering Scheme with Transmission Power Control for Sensor Networks[J]. Ieice Technical Report, 2005.

[19] Wang J, Yang L S, Zhao Y F. Research of Forestry Monitor Wireless Sensor Networks Cross-layer Power Control Based on Link Quality[J]. Radio Communications Technology, 2010.

[20] Sun Hongliang, Lu Hongbin. Measurement and Analysis of Environmental Impact on Wireless Link Quality[J]. China Science and Technology Expo, 2009(14):198-198.

[21] MIRJANA M, VALDIMIR V, VLADIMIR M. Fuzzy Logic and Wireless Sensor Networks-A Survey[J]. Journal of Intelligent \& Fuzzy Systems. 2014, 27(2): 877-890. 
[22] HUANG Y, DIAZ VH, SENDRA J. A novel topology control approach to maintain the node degree in dynamic wireless sensor networks [J]. Sensors. 2014, 14(3): 46724688. https://doi.org/10.3390/s140304672

[23] Soni V, Mallick D K. Fuzzy logic based multi-hop topology control routing protocol in wireless sensor networks[J]. Microsystem Technologies, 2018(7):1-13.

\section{Authors}

Huangshui Hu is an associate professor in Changchun University of Technology. He received his B.Sc. degree in 1997 from Jilin University, received his M.Sc. degree in 2005 from Jilin University, and received his Ph.D. degree in 2012 from Jilin University. His main research interests include wireless sensor networks and train communication networks.

Man Zheng is a graduate student in College of Computer Science and Engineering, Changchun University of Technology. She has received her B.Sc. degree in 2016(year) from Hubei University of Arts and Science. Her main research interests include wireless sensor networks and train communication networks.

Bangcheng Zhang is a professor in Changchun University of Technology. He received his B.Sc. degree in 1995, M.Sc. degree in 2004, Ph.D. degree in 2011 from Jilin University. His main research interests include mechatronics, complex system fault diagnosis and forecast and service robot.

Jinhui Qing is a graduate student in College of Computer Science and Engineering, Changchun University of Technology. He has received her B.Sc. degree in 2017(year) from Changchun University of Technology. His main research interests include wireless sensor networks and train communication networks.

Article submitted 05 April 2018. Resubmitted 24 June 2018. Final acceptance 10 julx 2018. Final version published as submitted by the authors. 